\title{
Supporting Information for "Spike Enabled Audio Learning in Multilevel Synaptic Memristor Array Based Spiking Neural Network"
}

\author{
Xulei $\mathrm{Wu}^{1,2}$, Bingjie Dang ${ }^{2}$, Hong Wang ${ }^{3}$, Xiulong $\mathrm{Wu}^{1}$, and Yuchao Yang ${ }^{2}$ \\ ${ }^{1}$ Anhui University \\ ${ }^{2}$ Peking University \\ ${ }^{3}$ Xidian University
}

September 10, 2021

\section{Authors}

Xulei $\mathrm{Wu}^{1,2}$, Bingjie Dang ${ }^{2}$, Hong Wang ${ }^{5}$, Xiulong $\mathrm{Wu}^{1, *}$, and Yuchao Yang ${ }^{2,3,4, *}$

${ }^{1}$ School of Electronics and Information Engineering, Anhui University, Hefei 230601, China.

${ }^{2}$ Key Laboratory of Microelectronic Devices and Circuits (MOE), School of Integrated Circuits, Peking University, Beijing 100871, China.

${ }^{3}$ Center for Brain Inspired Chips, Institute for Artificial Intelligence, Peking University, Beijing 100871, China.

${ }^{4}$ Center for Brain Inspired Intelligence, Chinese Institute for Brain Research (CIBR), Beijing, Beijing 102206, China.

${ }^{5}$ Key Laboratory of Wide Band Gap Semiconductor Technology, School of Advanced Materials and Nanotechnology, Xidian University, Xi'an, 710071, China

\begin{abstract}
Although speech recognition has been widely implemented in software neural networks, a hardware implementation based on energy efficient computing architecture is still missing. In this study, we have fabricated $\mathrm{W} / \mathrm{MgO} / \mathrm{SiO}_{2} / \mathrm{Mo}$ memristor array with multilevel resistance states, where the weights of the artificial synapses in the memristor array can be tuned precisely by voltage pulses. Based on the array, we have performed speech recognition in memristive spiking neural network (SNN) with improved supervised tempotron algorithm on TIDGITS dataset, demonstrating software-comparable accuracy for speech recognition in the memristive SNN. We envision that such memristive SNN can pave the way to building a bio-inspired spike-based neuromorphic system for audio learning.
\end{abstract}




\section{ToC Figure}

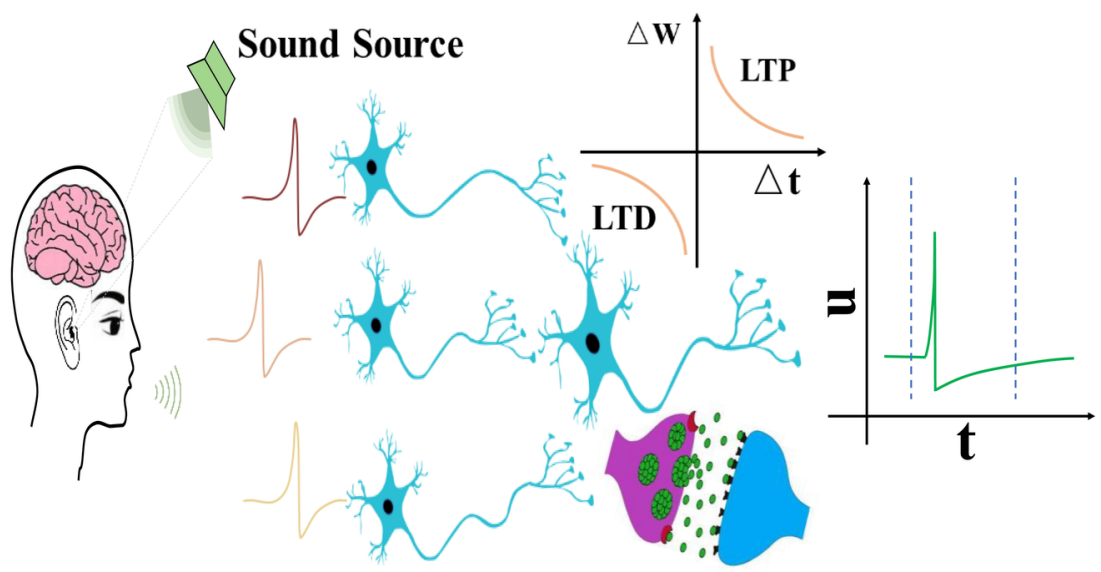

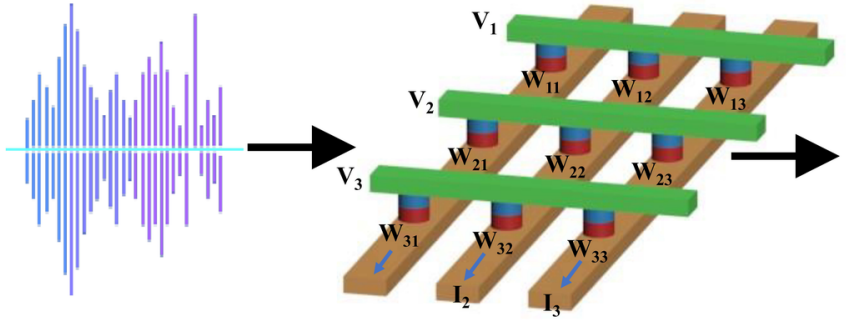

Audio Signals Memristor Crossbar Circuit

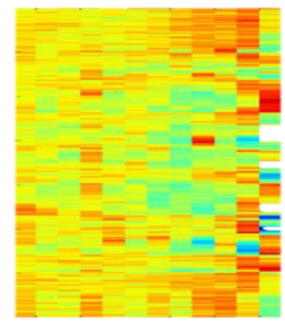

Classification

Here we report a $\mathrm{W} / \mathrm{MgO} / \mathrm{SiO}_{2} / \mathrm{Mo}$ memristor array with multilevel resistance states, where the weights of the memristor array can be tuned precisely by voltage pulses. Based on the array, we have performed speech recognition in memristive spiking neural network with improved supervised tempotron algorithm on TIDIGITS dataset, demonstrating software-comparable accuracy for speech recognition in the memristive spiking neural network.

\section{Supporting Information}

(a)

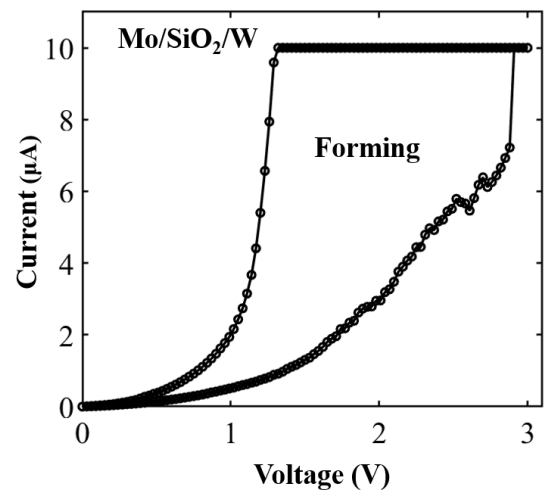

(b)

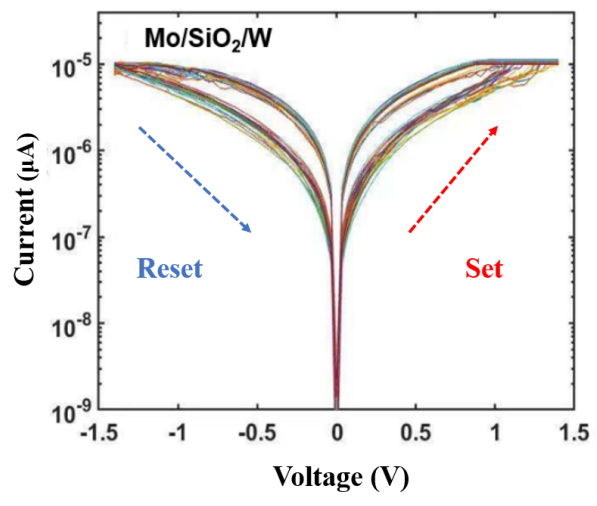


Figure S1.(a) The forming operation of the $\mathrm{Mo} / \mathrm{SiO}_{2} / \mathrm{W}$ device under the $+3 \mathrm{~V}$ DC sweeping voltages at room temperature. (b) The $I-V$ characteristics of the $\mathrm{Mo} / \mathrm{SiO}_{2} / \mathrm{W}$ device with $+1.45 \mathrm{~V} /-1.45 \mathrm{~V}$ write/erase voltages in 50 repeated DC sweeps, showing the resistive switching behaviors.

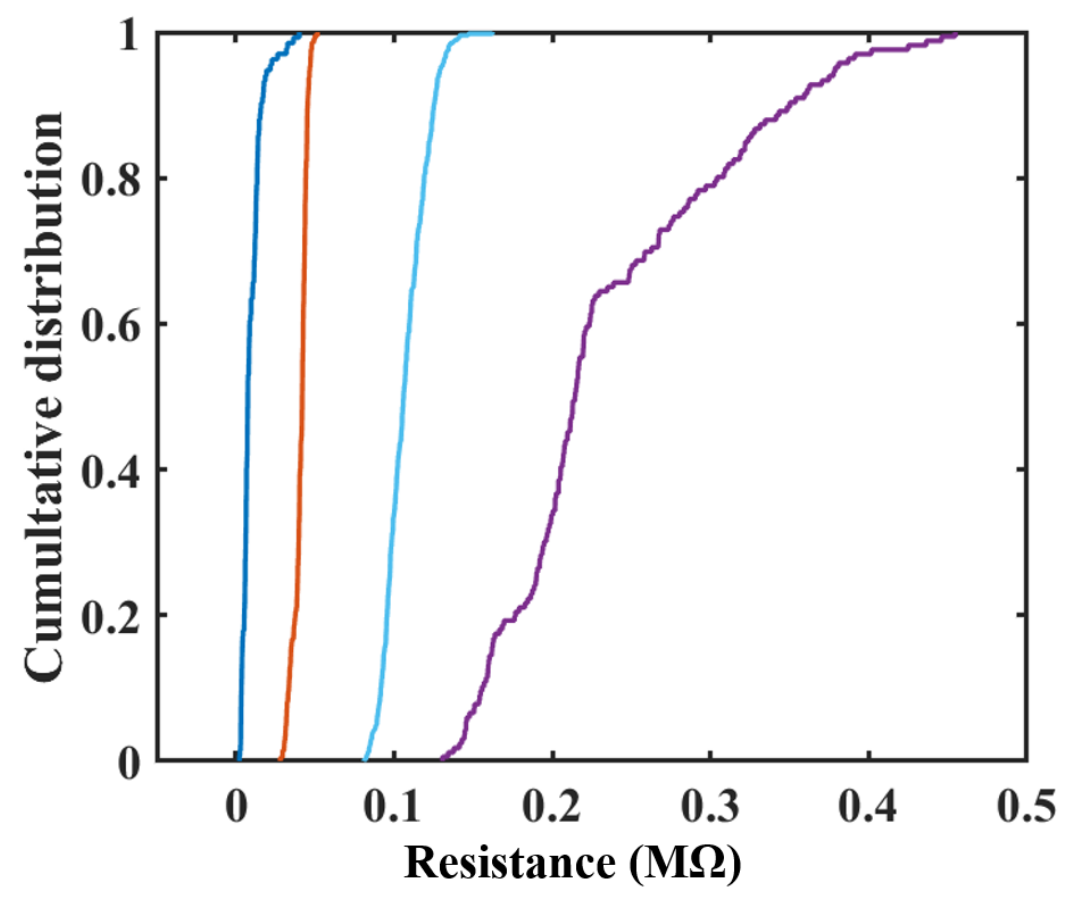

Figure S2. The cumulative distribution of resistance states of the $\mathrm{Mo} / \mathrm{SiO}_{2} / \mathrm{MgO} / \mathrm{W}$ devices. 


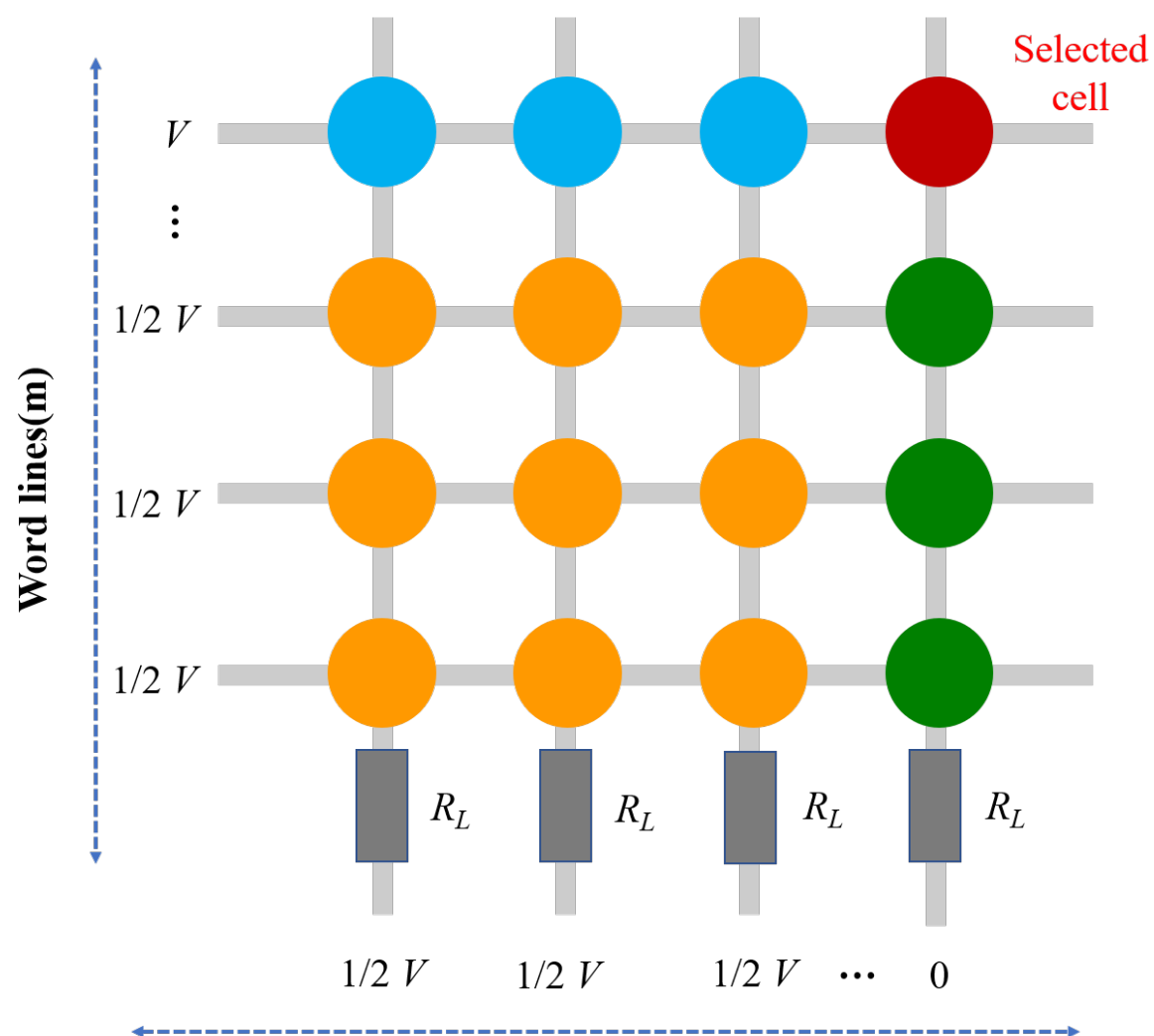

Bit lines(n)

Figure S3. The write/erase and read programming method in the memristors-array based on the one-half voltage $(\mathrm{V} / 2)$ bias scheme.

\section{(a)}

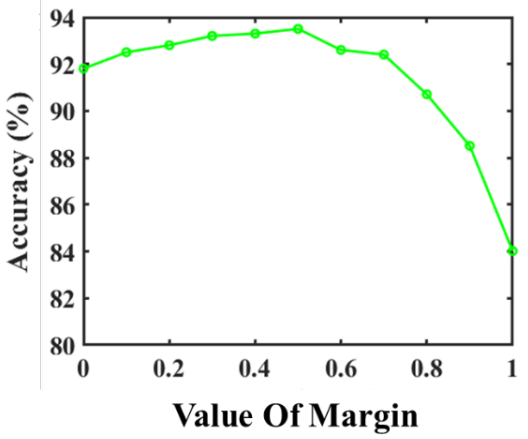

(b)

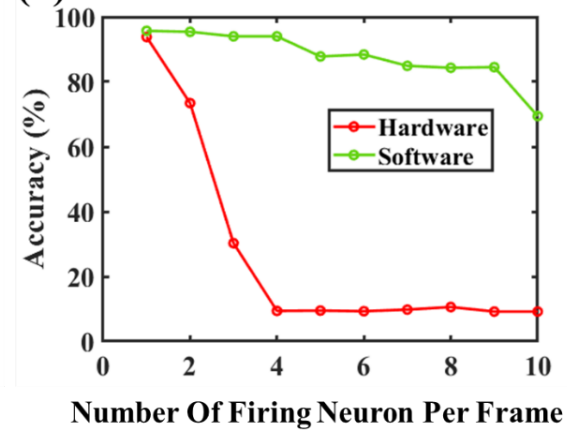

Figure S4. (a) The effect of the hard margin [?] on classification accuracy. For [?]=0, the modified learning rule is reduced to the classical Tempotron rule. (b) The effect of the number of activated output neurons in the SOM for each sound frame. 

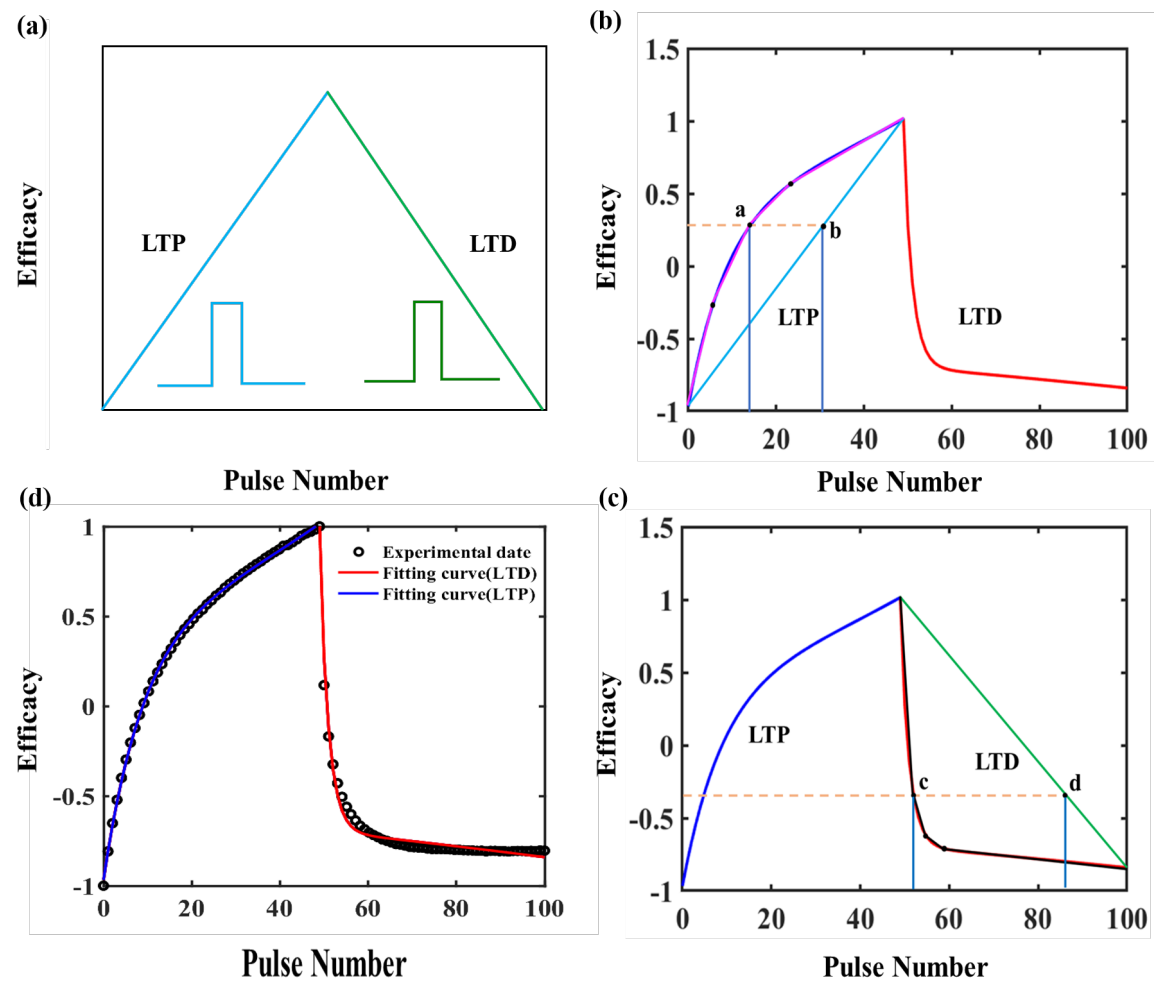

Figure S5. (a) The ideal and symmetric LTP and LTD. (b) Piecewise linear approximation of LTP. (c) Piecewise linear approximation of LTD. (d) The experimental data quantified and fitting curve of LTP and LTD.

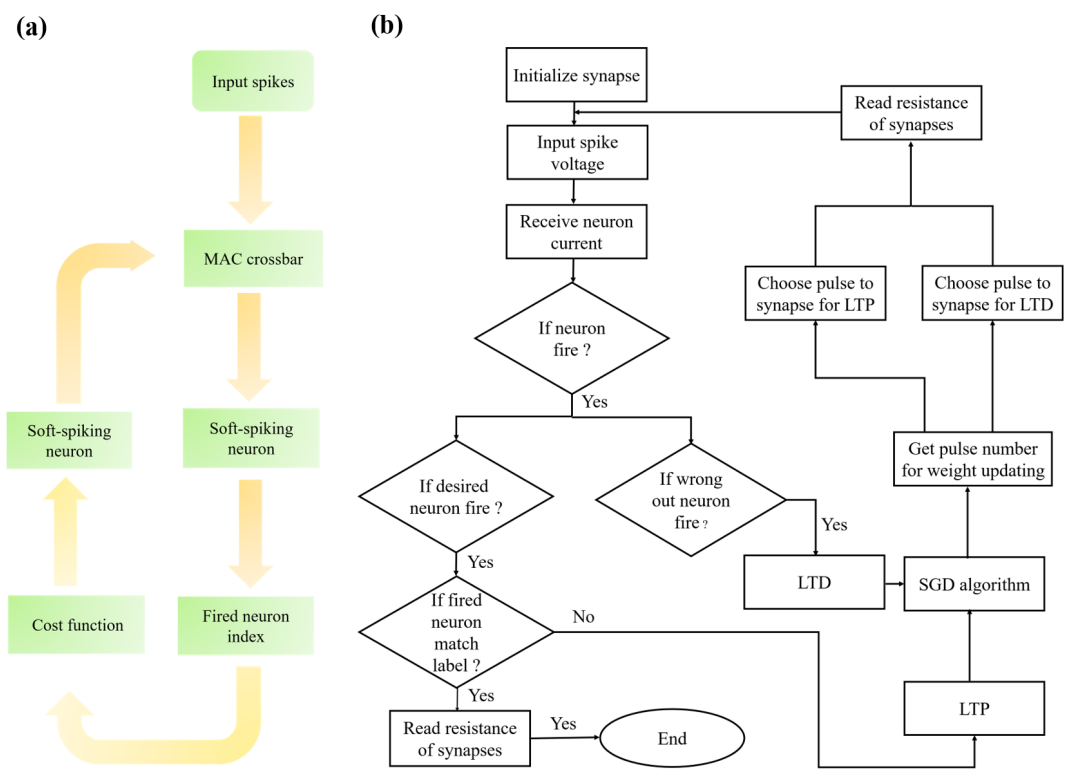

Figure S6. (a) Flow chart of the simulation process. (b) Flow chart of the training process. 


\section{Supplementary Movie1}

Rich media available at https://youtu.be/tmy9bfasjZU

\section{Supplementary Movie2}

Rich media available at https://youtu.be/y_WFbKq4Jw4

\section{References}

[S1] B. Dang, K. Liu, J. Zhu, L. Xu, T. Zhang, C. Cheng, H. Wang, Y. Yang, Y. Hao, R. Huang, APL Mater. 2019, 7, 071114.

[S2] Y. Chen, G.-Y. Jung, D. A. A. Ohlberg, X. Li, D. R. Stewart,J. O. Jeppesen, K. A. Nielsen, J. F. Stoddart and R. S. Williams, Nanotechnology 2003, 14, 462.

[S3] J. Fu, N. Gong, IEEE Journal on Emerging and Selected Topics in Circuits and Systems 2019, 9(2), 377.

[S4] Q. Duan, Z. Jing, X. Zou, Y. Wang, K. Yang, T. Zhang, S. Wu, R.Huang,Y.Yang. Nature Communications 2020, 11(1), 3399. 